\title{
Ideias Sobre Epistemologia e Pesquisa em Administração
}

\author{
Nome: Antônio Sérgio A. Fernandes ${ }^{1}$ \\ Universidade Federal da Bahia - UFBA \\ e-mail:asaferna@outlook.com
}

Nome: Marco Antônio C. Teixeira ${ }^{2}$

Fundação Getúlio Vargas -FGV-SP

e-mail: marco.teixeira@fgv.br

Nome: Alex B. F. M. do Nascimento ${ }^{3}$

Universidade Federal Campina Grande - UFCG

\section{Nome: João Martins Tude 4}

Universidade Federal Bahia - UFBA

\section{RESUMO}

Este ensaio, sob a forma de uma pensata, discute ideias com relação à epistemologia que envolve a instrumentalidade da pesquisa em administração. A pesquisa em administração é ampla, vasta, multidisciplinar, repleta de métodos e procedimentos que podem ser utilizados e a análise da produção do conhecimento por parte do pesquisador se torna um exercício intelectual acerca de como a investigação científica se torna um elo entre o pesquisador e a comunidade científica que ele está inserido, no caso aqui, da área de administração. Assumimos como condição prévia que a compreensão da administração em sua episteme torna o pesquisador consciente de que este se encontra diante de campo científico complexo e, portanto, o esforço instrumental e procedimental propriamente dito requer conhecimento epistemológico capaz de gerar pesquisas mais sólidas acerca da realidade observada dos fenômenos sociais que a área de administração se debruça a investigar.

Palavras chave: ensaio, ideias, epistemologia, investigação, pesquisa em administração.

\section{ABSTRACT}

The aim of this essay, in the form of a pensata, is to discuss ideas regarding the epistemology that involves the instrumentality of administration research. Administration research is broad, vast, multidisciplinary, full of methods and procedures that can be used, and the researcher's analysis on the production of knowledge becomes an intellectual exercise on how scientific research becomes a link between the researcher and the scientific community that he is inserted in, in this case, the administration area. We assume as a precondition that the understanding of administration in its episteme makes the researcher aware that it is facing a complex scientific field and, therefore, the instrumental and procedural effort itself requires epistemological knowledge capable of generating research, more solid about reality observed social phenomena that the administration area investigates.

Keywords: essay, ideas, epistemology, investigation, administration research.

[Submetido em 07-jan-2022 - Aceito em: 14-fev-2022 - Publicado em: 25-fev-2022]

${ }^{1}$ Graduação em Administração (UFBA) [(1994)], Mestrado em Desenvolvimento Urbano (UFPE) [(1998)], Doutorado em Ciência Política (USP) [(2003)].

${ }^{2}$ Graduação em Ciências Sociais (PUC-SP) [(1996)], Mestrado em Ciências Sociais (PUC-SP) [(1999)], Doutorado em Ciências Sociais (PUC-SP) [(2004)].

${ }^{3}$ Graduação em Administração (UESPI) [(2008)], Mestrado em Administração (UFRN) [(2012)], Doutorado em Administração (UFRN) [(2019)].

${ }^{4}$ Graduação em Administração (2005) [(UFBA)], Especialização em Met. e Didática do Ens. Superior (FSBB)[(2007)], Mestrado em Administração (UFBA)

[(2007)], Doutorado em Administração (UFBA) [(2010)]. 


\section{Introdução}

Um dos principais pontos que se faz presente quando pensamos mais profundamente com relação aos aspectos metodológicos em ciências humanas, sociais e sociais aplicadas são as bases epistemológicas que levam a uma concepção teórica vis-avis a definição e adoção de procedimentos e instrumentos de pesquisa propriamente ditos. Isso é crucial para que possamos compreender uma série de elementos importantes, que vão desde a escolha do viés dos casos a serem estudados, passando pela definição de amostras, bem como bases de dados, modelagens analíticas, indo até a indefinição ou indiferença aos critérios de validação interna e externa da pesquisa.

O objetivo deste ensaio, sob a forma de uma pensata, é desenvolver argumentos com relação à epistemologia que envolve a instrumentalidade da pesquisa em administração. Uma condição prévia assumida é que a compreensão da administração em sua episteme torna o pesquisador consciente de que este se encontra diante de campo científico complexo e, portanto, o esforço instrumental e procedimental propriamente dito requer conhecimento epistemológico capaz de gerar pesquisas, com bases sólidas acerca da realidade observada dos fenômenos sociais que a área de administração se debruça a investigar.

A solidez epistemológica torna o pesquisador fluente em qualquer metodologia instrumental, partindo do pressuposto do clássico King, Kehoane and Verba (1993) Social Inquiry Design de que não existem fronteiras rigorosas entre pesquisa qualitativa e quantitativa, o que de fato existem em ciências sociais são pesquisas com análise de dados e ou informações qualitativas, ou que utilizam procedimentos e métodos quantitativos e também pesquisas centradas em variáveis dependentes, com modelagens estatísticas, mas que ao mesmo tempo são também qualitativas, pois não prescindem da história, dos depoimentos, da observação participante, do estudo de casos e das reflexões teóricas mais amadurecidas. Nossa perspectiva aqui é que as ciências sociais aplicadas e em específico a administração possuem uma reflexão daquilo que é considerado a análise de como se explica cientificamente o fenômeno e o objeto, bem como a parte aplicada propriamente dita, ou seja, os instrumentos e procedimentos. As duas perspectivas não estão dissociadas, apenas ocupam papéis distintos na construção da pesquisa e do conhecimento em administração (Santos et al, 2009).

Ademais, com a normalização do produtivismo acadêmico inerente ao contexto contemporâneo, áreas de conhecimento ainda mais complexas das Ciências Sociais Aplicadas como Administração, e suas subáreas - possuem muita permeabilidade disciplinar, expectativa de contribuição tecnológica e processos de variabilidade muito marcantes, tornando-as cada vez mais difíceis de chegar ao preenchimento de lacunas efetivamente claras no seu rico universo cumulativo de conhecimento. Neste sentido, seguindo o que assinala Bertero (2011) experimentamos essa difícil situação em ciências sociais aplicadas e em administração em específico, que gira em torno de uma tensão existente entre a hegemonia da ciência positiva frente a reflexões dela mesma, como esta que se propõe aqui neste ensaio.

Acresce-se a isso a natureza reflexiva intelectual em si mesma de um ensaio, como considera Meneghetti (2011, p. 344):

"Os intelectuais por excelência usam o ensaio como forma para manifestar suas 
reflexões. Não é a reflexão daqueles que pensam ao escreverem, mas daqueles que escrevem após pensar e refletir. Só por este critério é possível perceber que são poucos os que realizam efetivamente um ensaio."

Ou seja, um ensaio é antes de tudo uma forma de reflexão intelectual que não consiste em sistematização baseada em cientometria (scientometrics) de ideias já escritas, mas um exercício de pensamento que é anterior e independente de qualquer caráter de compilação, ainda que algumas inferências lógicas se apoiem em ideias e hipótese ou pressupostos já enunciados. Basicamente, é um exercício de pensamento amplo no desenvolvimento de um tema (Meneghetti, 2011).

Este ensaio busca contribuir com um tema que até hoje é objeto de análises e por isso não diminui a chama de uma discussão que não quer calar até hoje na área. Por outro lado, tampouco queremos relatar de modo trivial algo profundo e valoroso para a área, ainda que sabido por experts, não possui visão muito precisa e uma única explicação que seja abrangente. Cremos que a profundidade e a amplitude do tema justificam a reflexão deste, sob vários ângulos, inclusive o que vamos tratar aqui. Isso é mais evidente quando assumimos que no campo dos estudos organizacionais, de fato, alguns conceitos são absolutizados, sendo incorporados ao saber pré-existente, pretendendo definir a realidade apesar dela (Faria, Maranhão, Meneghetti, 2013).

$\mathrm{O}$ ensaio está dividido em quatro partes contando com esta introdução. $\mathrm{Na}$ seção 2 procuraremos discutir o campo e o objeto de estudo em administração. A seção 3 traz tópicos de discussão sobre elementos metodológicos da pesquisa de Administração e a seção 4 traz considerações finais a guisa de conclusão.

\section{O Campo e Objeto de estudo em Administração}

A Administração é uma área bastante rica em termos de investigação social. Tendo como centro do seu objeto-problema a organização, ela traz uma riqueza epistemológica que sintetiza um caleidoscópio complexo de métodos e procedimentos de pesquisa, que por vezes tornam difícil situar sua episteme. Importante ressalvar que de acordo com Serva (2017), no Brasil, os estudos de administração possuem 8 dimensões epistemológicas importantes: 1) pragmatismo e práticas organizacionais; 2) estudos epistêmicos e filosóficos; 3) Racionalidade; 4) Problemática da instituição escolar e do ensino; 5) Administração pública; 6) História e organizações; 7) Abordagem decolonial; 8) Análise da inovação. Isso traz a disciplina da administração uma série de estudos dotados de mecanismos procedimentais muito dinâmicos, o que torna a disciplina de Administração muito complexa em termos epistemológicos. Na área de Administração Pública, por exemplo, como observam Valadares et al (2017), é bastante nítido o reflexo desse caráter multifacetado epistemologicamente da grande área de administração. Segundo os autores no contexto da administração pública no Brasil, a discussão de matrizes ontológicas e epistemológicas mostra a importância de compreensão do campo por um olhar multifacetado, frente às demandas e interlocuções entre Estado, Mercado e Sociedade.

Algo que importa na discussão sobre a epistemologia da investigação na área de administração é a diferença entre objeto e fenômeno. O objeto é uma derivação interpretativa do fenômeno por parte do pesquisador. Estabelecer esta diferença é fundamental para o pesquisador, pois por vezes há certa confusão na identificação desses 
dois conceitos. De acordo com Meneghetti (2011):

"O mais comum, no entanto, é atribuir um objeto de análise já no início do ensaio. Ocorre que o processo de análise só é possível na manifestação do objeto como fenômeno. O ponto central está na compreensão do fenômeno, levando em consideração que o fenômeno é aquilo que se apresenta; a separação entre essência e aparência é uma consequência natural. Por mais que o pensamento tente compreender e abarcar a totalidade da realidade, é fato que a consciência não consegue realizar esse empreendimento. Assim, conforme Marx afirma, se a essência fosse igual à aparência, a ciência inteira não existiria. Analogamente, se o objeto fosse igual ao fenômeno, não seria possível a formação da consciência. A compreensão do objeto requer escolhas de ordem epistemológicas. Elas não implicam cair no dogmatismo da epistemologia escolhida."

Uma das principais contribuições da Administração em termos teóricometodológicos e, portanto, epistemológicos também, é sua capacidade de possuir instrumentos que são capazes de produzirem uma melhor compreensão dos processos, sistemas e interações psicossociais entre pessoas e grupos, bem como, ao mesmo tempo, possuir também a natural vocação para intentar a proposição de novos modelos organizacionais envolvendo inovação e difusão destes modelos. Um dos mais importantes elementos em Administração é que ela é naturalmente uma ciência empírica, que se vale de engenhos inovativos diversos que acompanham o avanço tecnológico, quando ela mesma não é a própria propositora destes avanços tecnológicos. Isso lhe traz um status de ciência que possui um alto grau de perecibilidade de abordagem teórico-metodológica de seus fenômenos, algo que é inerente às ciências aplicadas, sendo a Administração uma das principais ciências sociais aplicadas.

Há duas décadas as pesquisas em ciências sociais aplicadas em grande parte das vezes vêm subestimando essas reflexões, o que causa na prática inúmeros problemas aos projetos de pesquisa e ao (a) próprio (a) pesquisador (a), sobretudo no que se refere à capacidade que o estudo obterá em termos de divulgação e/ou publicação em periódicos qualificados, capazes de contribuir com a descoberta, o debate e o avanço da ciência em uma determinada subárea do conhecimento. Este importante aspecto marca as ciências humanas e sociais no contexto contemporâneo dentro de sua busca incessante de estabelecer-se como campo de ciência centrada em dados e informações experimentais que se mostram capazes de refletir cada vez mais fortemente a realidade de um objeto que é marcadamente caracterizado pela variabilidade dos fenômenos observados. Isso leva ao retorno de uma discussão originária acerca da natureza do conhecimento em ciências sociais que vai do positivismo crítico à fenomenologia, passando pelo pósestruturalismo, entre outras importantes escolas de pensamento (Santana, 2008).

Além de todos esses elementos aqui apontados na área de Administração, importante ressaltar que isso é ainda bem mais evidente quando observamos sua permeabilidade disciplinar ao serem também observados os seguintes aspectos: 1) seu espaço fronteiriço é muito tênue com inúmeras áreas correlatas - Psicologia, Engenharia de Produção, Sociologia, Economia, Direito, entre outras e 2) sua natureza empirista devido ao fato de ser genuinamente Administração. Isso torna sua pesquisa muito suscetível a trabalhos bastante diversificados, chegando a um estado em que podemos considerar de modo parcialmente paradoxal de generalista. Evidente que isso não exclui 
o fato de haver verticalidade no tratamento de inúmeros temas importantes atuais, com centenas de objetos de estudo e unidades de análise. Diferentemente de áreas que possuem uma capacidade de isolar mais intensamente o objeto-problema, tal como é o caso em diversos campos disciplinares, tanto em ciências humanas e Sociais, como em Ciências Naturais e exatas, em administração os temas permeiam todas as subáreas de modo multidisciplinar e daí decorre sempre a preocupação epistemológica. A multidisciplinaridade é uma característica, portanto, de administração e analisar a epistemologia do seu campo disciplinar e, portanto, requer uma tentativa de realizar uma arqueologia de conceitos, temas e disciplinas que interagem nem sempre de modo claro.

Outro elemento de análise acerca da epistemologia da pesquisa em Administração é como se dá o processo de validação da pesquisa. Este processo compreende três momentos que incluem a validação prévia, validação interna e validação externa. A validação prévia corresponde ao processo inicial da pesquisa no que diz respeito à escolha adequada dos instrumentos e procedimentos de pesquisa previstos no projeto de pesquisa. A validação interna trata da etapa relacionada ao desenvolvimento da pesquisa propriamente dita. E a validação externa relaciona-se aos resultados gerados pela pesquisa. Originalmente a noção de validação da pesquisa é observada nos trabalhos caracterizados como pesquisa orientada por variável, que utilizam métodos quantitativos de investigação.

Neste sentido, a validação de pesquisas que utilizam métodos quantitativos, corresponde ao quão precisa é uma medida (categórica ou discreta) e, portanto, o quanto ela representa corretamente o conceito do tema-objeto que em estudo. A validação em estudos qualitativos é diferente, não necessariamente refere-se à semelhança entre as hipóteses extraídas de um conceito e suas medidas, mas ao nível de compreensão em que uma medida acurada representa o que se espera daquele tema-objeto. A garantia da validação começaria com a compreensão direta do que deve ser medido, sendo, portanto, um elemento detalhadamente exposto no projeto de pesquisa (HAIR Jr. et al., 2007). Para que uma pesquisa efetivamente possua validação estrita, é necessário que outro (a) pesquisador (a) deve (a) poder replicá-la metodologicamente, chegando a resultados próximos ou semelhantes. Nas pesquisas que utilizam metodologias qualitativas, seja, pesquisas qualitativas - pesquisa-ação, história oral, análise de conteúdo, pesquisa documental, entrevistas etc., ou análise de dados qualitativos (Pereira, 1999) - surveys, por exemplo, a validação assume um processo diferente, pois as escalas de mensuração ou não existem ou são construções abstratas (tipologias, categorizações etc).

Assim também é o caso das surveys, em que se podem utilizar ferramentas de expressão espacial como multidimentional scale (MDS) ou dendogramas, porém o mais importante seja em pesquisas qualitativas ou de análise de dados qualitativos é observar a qualidade da pesquisa, a confiabilidade dos dados e informações coletadas e sua relevância para a contribuir nas lacunas daquela tema-objeto de conhecimento. Seja, portanto, uma pesquisa quantitativa ou qualitativa, o que determina a validação desta é se de fato a pesquisa é capaz de medir factualmente o objeto-problema e se as inferências analíticas contidas em sua modelagem de explicação são lógicas e possuem resultados procedentes.

\section{Tópicos de discussão sobre elementos metodológicos em pesquisa de Administração}

Como exemplo, seguem alguns tópicos de discussão sobre elementos metodológicos 
em pesquisa de Administração que podem ser observados como elementos analíticos relevantes na melhor compreensão da produção do conhecimento em administração:

a) As fronteiras entre projeto acadêmico e profissional devem ser bastante tênues. Hoje, o mais importante na agenda política das mais variadas agências é a capacidade de gerar novos trabalhos de mestrado e doutorado que sejam capazes de dar retorno social. Vide a criação recente dos programas de doutorado profissional. Por vezes assistimos a uma confusão nessa questão (estudos tecnológicos como dissertações meramente acadêmicas e dissertações que deveriam ser meramente acadêmicas como estudos tecnológicos). Explicaremos melhor. Isso passa a ocorrer, sobretudo com a difusão ao final dos anos 1990, dos mestrados profissionais, onde o foco que se busca são estudos que representem uma contribuição tecnológica, que pode ser expressa numa dissertação ou em um projeto de intervenção. Por mais das vezes, como essa fronteira em muitos programas de pós-graduação ainda não se encontra muito bem definida, observase que grande parte dos estudos dos mestrados profissionais acaba se assemelhando aos trabalhos desenvolvidos nos mestrados acadêmicos. Ou seja, são trabalhos que tem destaque para teorizações e modelagens de explicação, mas sem que haja uma proposta mais concreta de qualquer protótipo ou novo processo gerencial prático, que represente efetivamente uma contribuição tecnológica. Em sentido contrário, também observamos por vezes que dissertações oriundas de programas acadêmicos, se apresentam com uma estrutura de trabalho tecnológico, onde valoriza-se pouco a teorização, e se investe pesadamente na construção de uma proposta de intervenção prática. Isso precisa ser melhor regulamentado nos programas de pós-graduação em Administração, pois acaba causando dissonâncias entre proposta do candidato a mestrado e a expectativa da mesa examinadora.

b) A elaboração de uma pesquisa acadêmica propriamente dita requer a existência de um construto teórico mínimo. Portanto, isso exige do pesquisador muito esforço analítico, não basta ter um modelo baseado em variável dependente, ou uma etnografia bem estruturada. Em geral, o teste numa pesquisa acadêmica de Administração busca descrever e estabelecer causalidades. Isso é o plus ou a "arte" do pesquisador. Por vezes, também observamos, sobretudo em teses de doutoramento, um trabalho tímido neste ponto. $\mathrm{O}$ candidato apenas reproduz as categorias de um autor ou um conjunto de autores de ponta e as adapta construindo variáveis que se encaixem (fit) no trabalho. Por vezes observamos a existência de pouca preocupação em uma discussão e construção de um modelo teórico por parte do pesquisador, que seja capaz de dialogar com a teoria ou as teorias e conceitos que ali estão sendo abordados. Isso ainda é um vício constante na academia da Administração. Assim, teses de doutoramento, e até pesquisas, já de pesquisadores seniores, podem apresentarem-se sem qualquer elemento novo em termos teóricos, apenas a reprodução das categorias dos autores que tratam do conceito que se quer aplicar. Como Administração faz parte das Ciências sociais aplicadas e, portanto, é uma disciplina tecnológica, por vezes a aplicação direta das categorizações de ponta internacionais não apresentam os resultados, sejam por modelagem estatística de variáveis, seja por estudos de caso, porque basicamente, desafiam o princípio da variabilidade de fenômenos tecnológicos. O que é explicável no caso de alguns países não necessariamente é líquido e certo em outros países. Sempre foi um problema em Administração, os processos de difusão de tecnologias e conceitos gerenciais. Por exemplo, destacando um tema bem atual e quase clássico dentro da subárea de administração pública: reformas administrativas que deram certo num determinado lugar e que geraram muitos estudos e debates em âmbito global, não necessariamente 
tiveram êxito na sua implementação quando replicada em outros países sem que se considerassem as devidas particularidades. Ou seja, é sempre muito difícil observar o isomorfismo dos processos gerenciais quando se trata de resultados de pesquisas em Administração.

c) Modelos estatísticos ou estudos de casos comparados são fundamentais, mais que isso, a abordagem multimétodo é hoje a mais indicada, não há como fugir disso, tem-se que ter habilidades metodológicas diversas, o que se busca ver em uma pesquisa é a lacuna preenchida, o novo. Chegar ao novo é algo demorado, na maior parte das vezes nem sempre se chega, apenas chega-se perto. Por exemplo, um estudo de caso em Administração, pode utilizar diversas metodologias de pesquisa - pesquisa de survey (ou seja, análise de dados qualitativos), pesquisa qualitativa propriamente dita (ou seja, história oral, análise de conteúdo, narrativas analíticas entre outras). Ou pode ser um caso que utiliza metodologia orientada por variável, onde o pesquisador constrói sua base de dados empíricos, através do apontamento de registros e utiliza estatística inferencial para desenvolver sua análise. De fato, no contexto atual, a fronteira é muito tênue entre o que no passado se chamava de abordagem qualitativa e abordagem quantitativa. $\mathrm{O}$ que se observa no momento é a prevalência de mixed method studies (Caldas, 2003; Creswell, 2002).

d) Outro ponto importante diz respeito à formação do pesquisador no contexto brasileiro, seja em universidades públicas (federais e estaduais), bem como escolas de negócios. É importante que a formação básica que vem sendo oferecida graduação seja mais voltada para formar um pesquisador júnior, capaz de saber instrumentos e procedimentos mais elementares de pesquisa, pois só assim conseguiremos ter o celeiro para os programas de pós que são oriundos da graduação. $\mathrm{Na}$ graduação é importante que o aluno (a) adquira ao longo do curso conhecimentos prévios do que se trata uma pesquisa em termos mais gerais. Programas de Iniciação Científica (PIBICs), bem como a existência centros ou núcleos de pesquisas liderados por professores que absorvam alunos da graduação em seus projetos, além de outras iniciativas de estímulo ao ingresso do graduando na pesquisa acadêmica, podem desenvolver papeis que são cruciais para uma formação básica mais rigorosa do jovem pesquisador. Esse é um problema notório para uma área aplicada como Administração e suas subáreas. Em Administração, sobretudo do ponto de vista curricular há uma proposta geral voltada para a formação visando uma atividade ou atuação profissional e não a formação de um pesquisador. Porém, talvez essa visão esteja míope, pois nada melhor para um excelente profissional se ele consegue diagnosticar e propor mudanças, alicerçado no caminho que a investigação científica lhe propõe - definição do objeto-problema, estabelecimento de objetivos, metodologia adequada de abordagem e inferências analíticas sólidas e baseadas na literatura clássica e de ponta que trata do problema em tela.

e) $\quad \mathrm{Na}$ pesquisa, a verticalidade dos temas deve ser perseguida, e uma das tensões do (a) pesquisador (a) de Administração é ao mesmo tempo ter um tema sendo verticalmente pesquisado e atender à generalidade dos demais temas que necessariamente terá interlocução ao longo da sua carreira nos diferentes momentos de suas atividades de pesquisa. Isso diz respeito especificamente aos pesquisadores seniores em suas orientações aos alunos de mestrado e doutorado e também as parcerias em estudos de temas adjacentes ao que o pesquisador trabalha de modo mais focado. Talvez em áreas das chamadas hard sciences como Física, Química, Biologia, Medicina, Engenharia, entre outras, a questão do foco mais preciso de pesquisa durante toda a vida de trabalho do pesquisador seja algo mais coerente. Entretanto em Ciências Humanas e 
Sociais Aplicadas, e especificamente em Administração, ao longo do tempo, as interseções com outros temas é algo que se mostra naturalmente e por isso a capacidade do pesquisador de Administração deve ser no sentido de manter a tensão entre seus projetos específicos e projetos adjacentes ao foco de pesquisa que este tem ao longo da carreira. Isso requer uma maior erudição do pesquisador, pois além de seu tema-objeto, as parcerias a temas adjacentes a seu tema-objeto específico o ajudam a consolidar uma área ou subárea de estudos importantes.

\section{Considerações Finais}

Após o desenvolvimento de alguns argumentos acerca da epistemologia da administração e sua influência na instrumentalidade da pesquisa, o que se pode inferir a guisa de conclusão é que de fato, o que vai contar mesmo metodologicamente na pesquisa em Administração e suas subáreas, é a riqueza da experiência de pesquisa. Seja qual for a fase em que o (a) pesquisador (a) esteja na formação (graduação, mestrado e doutorado), ou já como pesquisador (a) profissional, que busca contribuir de modo mais enfático e cada vez mais rigoroso metodologicamente com o fenômeno, bem como temaobjeto que abraçou. Torna-se importante isso, pois no mais das vezes os pesquisadores, estejam em formação acadêmica ainda, ou já em estágio sênior da carreira, desenvolvem bons projetos, mas sem refletir aquilo que está sendo estritamente tratado nos seus estudos. Isso pode levar a incorrem em juízos de valor que não trazem nenhum tipo de resposta ou nova pergunta analítica consistente e para além dos modelos formais. A formalização é uma etapa obrigatória de um trabalho de pesquisa, mas, como já dissemos ao longo deste ensaio, a administração, por se tratar de uma ciência social aplicada, requer análise, senso crítico, precisão e acurácia inferencial. Sem isso, os pesquisadores em Administração, de maneira mais geral, correm o risco de apenas replicarem modelos realizando testes sem solidez mais profunda.

\section{Referências}

BERTERO, Carlos Osmar. Réplica 2 - o que é um ensaio teórico? Réplica a Francis Kanashiro Meneghetti. Rev. adm. contemp., Curitiba, v. 15, n. 2, p. 338-342, Apr. 2011

Available from <http://www.scielo.br/scielo.php?script=sci_arttext\&pid=S141565552011000200012\&lng=en\&nrm=iso $>\quad$ access $\quad$ on $\quad 10$ July 2020. https://doi.org/10.1590/S1415-65552011000200012.

CALDAS, Miguel Pinto. Research design: qualitative, quantitative, and mixed methods approaches. Rev. adm. contemp., Curitiba , v. 7, n. 1, p. 223, mar. 2003 . Disponível em <http://www.scielo.br/scielo.php?script=sci_arttext\&pid=S141565552003000100015\&lng=pt\&nrm=iso>. acessos em 10 jul. 2020. https://doi.org/10.1590/S1415-65552003000100015.

Creswell, John. Research Design: Qualitative, Quantitative, and Mixed Methods Approaches. 2. ed. London: Sage Publications, 2002.

FARIA, José Henrique de; MARANHAO, Carolina Machado Saraiva de Albuquerque; MENEGHETTI, Francis Kanashiro. Reflexões epistemológicas para a pesquisa em administração: contribuições de Theodor W. Adorno. Rev. adm. contemp., Curitiba , v.
$17, \mathrm{n}$.
$6, \mathrm{p}$.
642-660,
Dec.
2013
Available
from 
$<$ http://www.scielo.br/scielo.php?script=sci_arttext\&pid=S1415-

$65552013000600002 \& \operatorname{lng}=\mathrm{en} \& \mathrm{nrm}=\mathrm{iso}>. \quad$ access on 10

July 2020. https://doi.org/10.1590/S1415-65552013000600002.

HAIR, J. F. P. J.; Babin, B.; Money, A. H.; Somouel, P. Fundamentos de Métodos de Pesquisa em Administração. São Paulo: Bookman, 2007. 471f.

KING Gary; KEOHANE, Robert O.; VERBA, Sidney (1994). Designing Social Inquiry. Scientific Inference In Qualitative Research. Princeton: Princeton University Press, 1994.

MENEGHETTI, Francis Kanashiro. O que é um ensaio-teórico? Rev. adm. contemp., Curitiba, v. 15, n. 2, p. 320-332, Apr. 2011. Available from $<$ http://www.scielo.br/scielo.php?script=sci_arttext\&pid=S1415-

65552011000200010\&lng=en\&nrm=iso $>$. access on 2020. https://doi.org/10.1590/S1415-65552011000200010

PEREIRA, Júlio César R. Análise de Dados Qualitativos: Estratégias Metodológicas para as Ciências da Saúde Humanas e Sociais. São Paulo: EDUSP, 1999. 156p.

SANTANA, P. E. A. Uma Breve Análise Didática dos Métodos Científicos Positivismo, Materialismo Histórico e Fenomenologia. Revista Cesumar - Ciências Humanas e Sociais Aplicadas, v. 13, n. 1, p. 25-35, jan./jun. 2008.

SCUSSEL, Fernanda Bueno C. Poder, Paradigmas e Domínio na Pesquisa em Marketing no Brasil: Uma Análise Da Produção Nacional da Disciplina a Partir das Matrizes Epistêmicas. RAEP - Administração: Ensino e Pesquisa Rio de Janeiro v. 18 no 3 p. 518 557 set-dez 2017.

SANTOS, Reginaldo Souza; RIBEIRO, Elizabeth Matos; SANTOS, Thiago Chagas Silva. Bases teórico-metodológicas da administração política. Rev. Adm. Pública, Rio de Janeiro, v. 43, n. 4, p. 919-941, Aug. 2009 . Available from

$<$ http://www.scielo.br/scielo.php?script=sci_arttext\&pid=S0034-

$76122009000400008 \& \operatorname{lng}=\mathrm{en} \& \mathrm{nrm}=\mathrm{iso}>. \quad$ access $\quad$ on

2020. https://doi.org/10.1590/S0034-76122009000400008.

SERVA, Maurício. Epistemologia da administração no Brasil: o estado da arte. Cad. EBAPE.BR, Rio de Janeiro , v. 15, n. 4, p. 741-750, Dec. 2017 . Available from $<$ http://www.scielo.br/scielo.php?script=sci_arttext\&pid=S1679-

39512017000400741\&lng=en\&nrm=iso> access $\quad$ on $\quad 10$ July 2020. https://doi.org/10.1590/1679-395173209.

VALADARES, Josiel L.; Pinto, Lauísa B.; Vilas Boas, Ana Alice; Brito, Mozart José. Administração pública sob a ótica dos estudos críticos: reflexões, interlocuções e tendências. Administração Pública e Gestão Social, 9(1), jan-mar. 2017, 31-42. 